\title{
Conjugated Polymer Nanoparticles
}
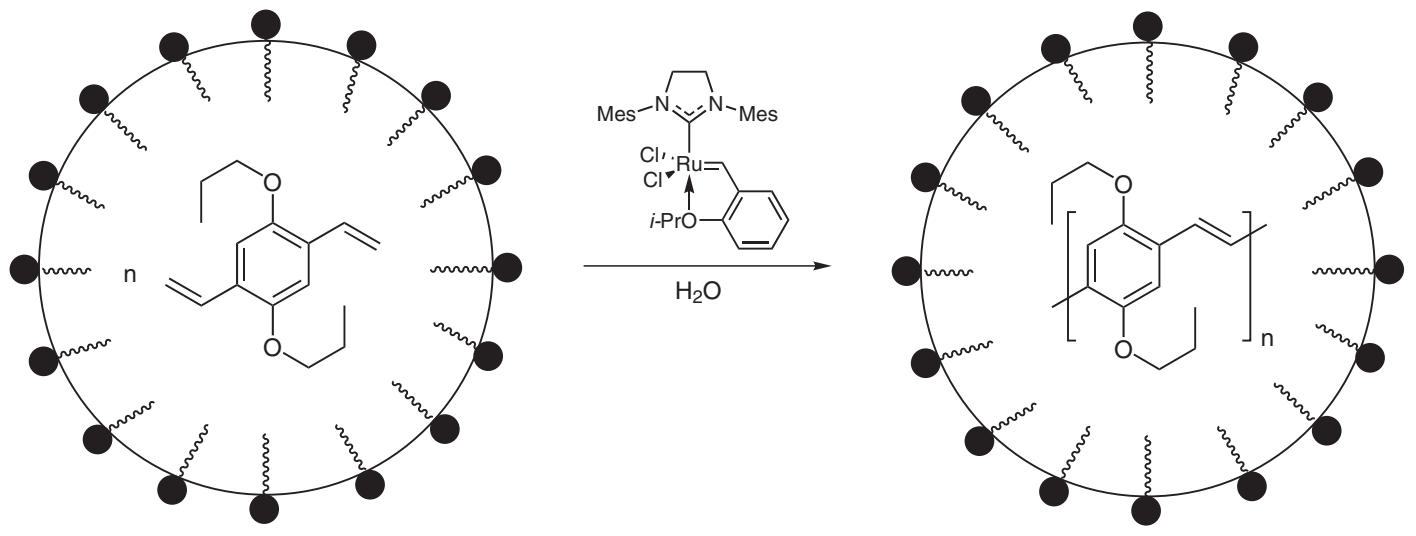

Key words

conjugated polymers

metathesis

nanoparticles

Significance: Nanoparticles of a conjugated poly(phenylene vinylene) (PPV) were synthesized by step-growth polymerization of a divinyl monomer in an aqueous emulsion. Model studies in toluene revealed that the Hoveyda-Grubbs metathesis catalyst afforded the highest yield (>95\%) of moderately high molecular weight PPV (Mn 2400 $\mathrm{g} / \mathrm{mol}$ ); additionally, the optimal monomer/catalyst ratio was also determined. Using these conditions, approximately $230 \mathrm{~nm}$-sized PPV nanoparticles were synthesized within surfactant-stabilized toluene microemulsions in water.
Comment: Dispersions of conjugated polymer nanoparticles have been shown to possess superior processability over simple polymer solutions (Scherf et al. Nat. Mater. 2003, 2, 408). Moreover, nanoparticles open the door to using conjugated organic polymers in a host of bioimaging applications. In this paper, the authors present a unique, 'bottom-up' method of synthesizing PPV nanoparticles. Whereas it is most common to form conjugated polymer nanoparticles by crosslinking individual polymer chains, the authors perform the reverse by growing polymer chains within a micelle. 